\title{
Improvement of Inertial Object Tracking for Low-cost Indoor-navigation with Advanced Algorithms
}

Haid, Markus; Günes, Ersan; Chobtrong, Thitipun; Kamil, Mustafa; Münter, Moritz

Competence Center for Applied Sensor Systems (CCASS), Hochschule Darmstadt, Birkenweg 8 , 64295 Darmstadt, E-Mail: markus.haid@h-da.de, Tel.: 0170 1670205, Fax: 0615116 8930, www.ccass.h-da.de

\section{Themenschwerpunkt:}

C8 Autonome Systeme, Robotik

\section{Abstract:}

To improve the performance of INS, the Competence Center for Applied Sensor Systems (CCASS) has successfully designed a low-cost INS with 6 accelerometers and 6 gyroscopes. The accuracy of this object-tracking system has been dramatically improved by Shaping-filter and Sensor-error model. According to the application of this navigation system, a pattern recognition algorithm will be developed to improve the stability and accuracy of the INS. This recognition algorithm is based on the Bayesian decision method, because it is a parametric technique with benefits of simplicity.

Our developing application has finite start and stop points. In other words, the pattern of the movement might possibly be recognized with pattern recognition algorithms. Then, the position will be estimated based on the result of the developing pattern recognition module and the navigation module compensated with the error module. Moreover, this pattern recognition module might be successfully integrated with the error module, which is based on Kalmanfiltering. It might possible to use the result from the pattern recognition as a reference signal, as well as the reset point of the INS system.

\section{Keywords}

Kalman filter, IMU, Indoor-navigation, Error model, Inertial navigation, Position estimation

\section{Introduction}

In recent years, quality control is one of the major focuses in automotive industries. Most processes in automotive manufacturing have been carefully observed and tested to ensure the quality of the end products. However, many automotive parts still have been assembled without any quality control process. For instance, all bolts of automotive parts should be fastened in the right position, and also in the right order. This manufacturing process remarkably depends on operators. Therefore, some mistakes might occur by human error, such as missing bolts or fastening a bolt in a wrong position. To prevent these problems and improve the quality of automotive assembly, the system which is able to track the location of the tool-tip location, while fastening the bolts, is required.

To develop a tool-tip tracking system using a low-cost inertial measurement unit (IMU), the boltidentification algorithm has been constructed for supporting the project "INSCHRAV". This identification algorithm is integrated in an intelligent screwdriver to use it in an automotive production line, such as engine assembly and air-bag assembly. With WIFI communication, the information from the intelligent screwdriver will be continuously sent to the monitoring system for checking the position and the order of fastened bolts.

A low-cost IMU is micro-electromechanical systems (MEMS) composed with three accelerometers and three gyroscopes that measure the linear acceleration and angular velocity in 3-axis of the body-frame [1]. Theoretically, the attitude of an object attached with an IMU is able to be numerically estimated by integration of angular velocity signals, and the position of 
the object is estimated by double integration of acceleration signals. However, the major problem of a low-cost IMU is stochastic sensor drift. The error from attitude and position estimation using IMU increases over time dramatically, because of the accumulated error from the integration [2].

To eliminate the effect of sensor drift from attitude estimation, Kalman filter (KF) and variants of Kalman filter is well-known technique to find the optimal attitude from the signals of a low-cost IMU. Foxlin applied extended Kalman Filter (EKF) based on the separated bias Kalman filter method from Friedland in [3], [4]. However, the yaw estimation of this method is depended on the signals from magnetometers as many studies in [5] - [9]. For automotive assembly line, there are many metallic objects that distort the magnetic field. Therefore, using magnetometers for attitude estimation might lead to the inaccuracy of the system. Another problem of EKF, it is well-known that the behavior of EKF is unpredictable, even though, it often can be used successfully [11].

Instead of EKF, other studies, such as in [12], successfully developed attitude estimation algorithms based on unscented Kalman Filter (UKF). Although the mathematic model of UKF is simpler than EKF, because of it is not necessary to calculate Jacobian matrices [13], the computation time of UKF is greater than EKF, and it is not suitable for real-time applications. Interestingly, Rehbinder and $\mathrm{Hu}$ successfully developed the attitude estimation based on switching Kalman Filter [11]. Importantly, the mathematic model of their work is simply and based on complementary Kalman filter and the switching algorithm in [14]. There is the switching algorithm for selecting between static mode and dynamic mode. This technique is really practical for eliminating the effect of lateral acceleration while the object is moving. However, the output of this attitude estimation is just only the optimal roll and pitch angles.

Without global positioning systems (GPS), there is a few of successful algorithms to estimate the position of an object using IMU. In [18], the authors developed the error model to generate the optimal error from the result of their experiments. Similarly, Hegrenses et al. generated the error module based on KF to compensate the error from position estimation using IMU [19] (Hegrenses et al., 2007). With signals from redundant accelerometers and gyroscopes, Haid successfully applied error module based on KF for one-dimensional position estimation in (Haid, 2004). Interestingly, these techniques are practical when the dynamic model is lack in observation or using IMU without other reference sensors.

Won et al. applied a position sensor to improve the performance of position estimation based on $\mathrm{KF}$ in [20]. With help of Fuzzy logic algorithms, the position of a fastening tool is successfully tracked with their system. However, using a position sensor is inconvenient in the automotive production line, because the workspace of this system is limit by the wire length of the position sensor.

Unlike other studies, Vissiere et al. developed a KF technique using the magnetic disturbance in observation model to compensate the error of the velocity estimation [21]. Importantly, the performance of this method is depended on the model of magnetic field disturbances. Based on Maxwell's equation, the mathematic model of this technique is remarkably complicated and difficult to implement in real applications.

This paper presents a position estimation based on complementary Kalman filter (CKF) and shaping filter for indoor applications which the movement of the tracking object is definite in patterns. Note that lower-case bold letters, Greek or Roman, denote vectors, and upper-case bold letters denote matrices.

\section{Overview of tool-tip tracking algorithms}

There are four main algorithm modules as shown in Figure 1, the initialize module, the attitude estimation module, the position estimation module and the bolt-identification module. In brief, 
the initialize module determines the initial position of the IMU, $\mathbf{r}_{o b, 0}$, and the initial orientation $\mathbf{q}_{n, 0}^{b}$ of the intelligent screwdriver by using the IMU and ultrasound sensors. From the initial position to the first bolt-position, the information of the optimal attitude $\hat{\mathbf{q}}_{n}^{b}$ is estimated by the attitude estimation module. Then, the position estimation module determines the optimal position of the tool-tip $\hat{r}_{t i p}$. Finally, the bolt-identification module estimates the recognized position $\overline{\mathbf{r}}_{r \varepsilon g}$ of the fastened bolt. $\mathrm{s}$

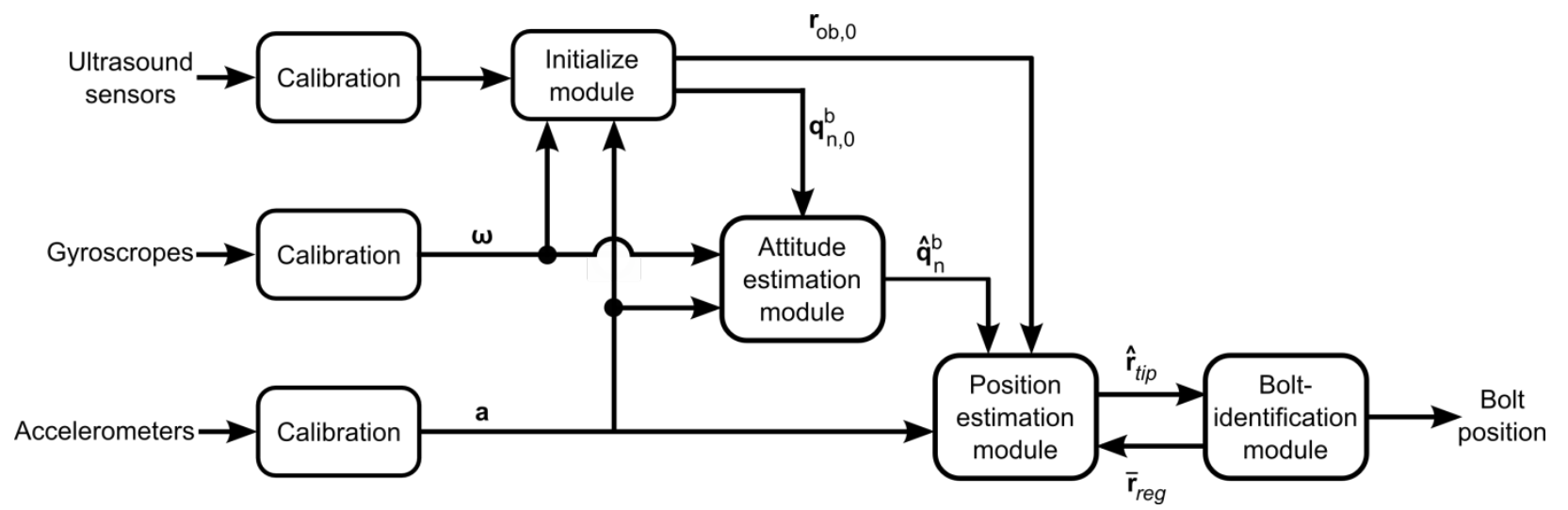

Figure 1: Overview of tool-tip tracking algorithms

There are two major components in the position estimation module, as shown in Figure 2. One of the main components is the navigation algorithm which estimates the position of an object by using the accelerometer signals and an optimal attitude $\hat{\mathbf{q}}_{n}^{b}$ from the attitude estimation module. Another main component is the error model based on complementary Kalman filter (CKF). To estimate the position of an object, the measurement signals from IMU (the acceleration and angular velocity signals) are pre-processed. After that, the navigation algorithm determines the predicted position $\overline{\mathbf{s}}$. Simultaneously, the error model as shown in Section 3, estimates the optimal position error $\boldsymbol{\Delta} \hat{\mathbf{e}}_{s}$. The optimal position $\hat{\mathbf{s}}$ is determined by compensating the predicted position with the optimal position error. Finally, the optimal tool-tip position $\hat{\mathrm{r}}_{\text {tip }}$ is computed by the optimal attitude and the optimal position.

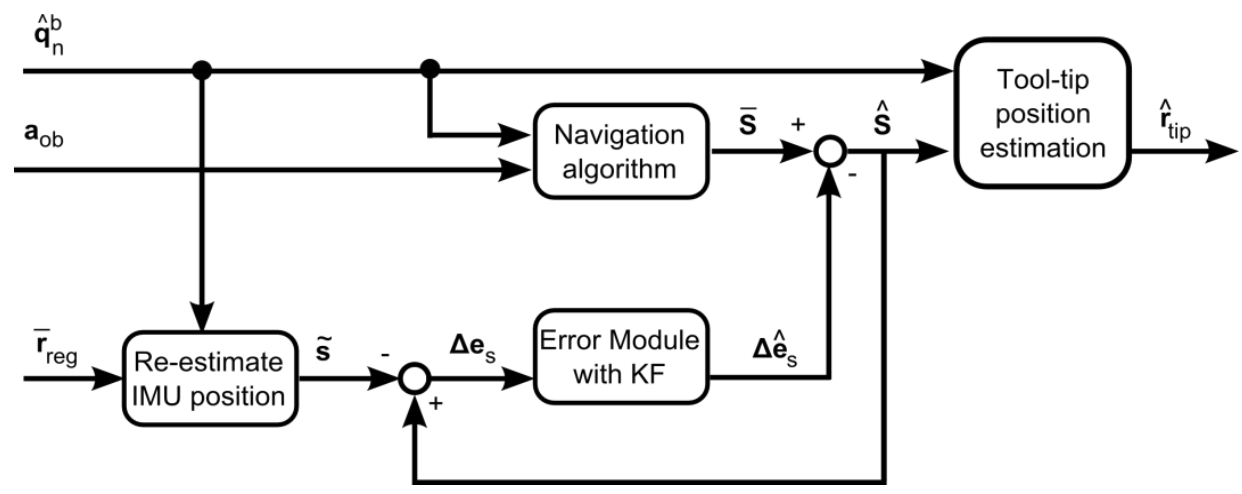

Figure 2: position estimation module

\section{Complementary Kalman filter}

In brief, Kalman filter is a recursive algorithm based on a stochastic model for estimating the optimal state, in sense of least square error under circumstances, at time $k$ by the the previous state (at time ${ }^{k-1}$ ). A dynamic system can be modeled by a $n$-by-1 state vector $\mathbf{x}$ obeying a discrete-time evolution equation as in $\mathrm{Eq}(1)$, where $\mathbf{F}$ is an $n$-by- $n$ state transition matrix, $\mathbf{G}$ is an $n$-by- $p$ matrix and $\mathbf{u}$ is a $p$-by- 1 vector of system inputs, and $\mathbf{w}$ is an $n$-by- 1 process noise vector with covariance $\mathbf{Q}_{k-1}$. 
$\mathbf{x}_{k}=\mathrm{Fx}_{k-1}+\mathbf{G u}_{k-1}+\mathbf{w}_{k-1}$

To observe a system, the measurement vector $\mathbf{z}$ is the indirect measurements of the state vector which is available at each time $k$. This measurement vector is defined as an $m-$ by-1 as in Eq (2), where $\mathbf{H}$ is an $m$-by- $n$ system observation matrix, and $\mathbf{v}$ is an $m-b y-1$ measurement noise vector with covariance $\mathbf{R}_{k}$.

$\mathbf{z}_{k}=\mathbf{H x}_{k}+\mathbf{v}_{k}$

One form of the discrete-time Kalman filter, shown in Eq (3), is applied for this attitude estimation,

$\widehat{\mathbf{x}}_{k}=\mathbf{F} \widehat{\mathbf{x}}_{k-1}+\mathbf{G} \widehat{\mathbf{u}}_{k-1}+\mathbf{K}_{k}\left(\mathbf{z}_{k}-\overline{\mathbf{z}}_{k}\right)$

where the Kalman gain matrix $\mathbf{K}$ is determined from the estimation error covariance matrix $\mathbf{P}$ as in Eq (4).

$\mathbf{K}_{k}=\mathbf{P}_{k} \mathbf{H}^{T}\left[\mathbf{H P}_{k} \mathbf{H}^{T}+\mathbf{R}_{k}\right]^{-1}$

In Eq (5), the estimation covariance matrix $\mathbf{P}$ is updated by the Ricatti equation:

$\mathbf{P}_{k}=\mathbf{F}\left[\mathbf{I}-\mathbf{K}_{k} \mathbf{H}\right] \mathbf{P}_{k-1} \mathbf{F}^{T}+\mathbf{Q}_{k}$

The predicted measurement vector, $\overline{\mathbf{z}}$, is computed from the previous state as in Eq (6).

$\mathrm{z}_{k}=\mathrm{HF}_{\mathrm{k}-1}$

Importantly, the term $\mathbb{Z}\left(z \rrbracket_{k}-\bar{z}_{k}\right)$ in Eq (3) is the correction term of Kalman filter, which can be observed the behavior of the Kalman filter [16].

While most of the authors above used a Kalman filter to directly estimate the position and it's derivatives, it is common in inertial navigation systems to instead use a complementary Kalman filter which operate only on the error in position estimation. This technique dramatically reduces the complexity of the model, and also improves the stability of the system when the KF model fails [15].

As Figure 2, the acceleration vector $\mathbf{a}_{o b}$ defined in Eq (8), is measured by the accelerometers, where $a_{o b, i}$ is the acceleration in $i$-axis of body-fixed frame.

$\mathbf{a}_{o b} \triangleq\left[\begin{array}{lll}a_{o b, x} & a_{o b, y} & a_{o b, z}\end{array}\right]^{T}$ 
Then, the navigation algorithm estimates the predicted position $\overline{\mathbf{s}}$. To compute the optimal position, the error model is designed to estimate the optimal position error $\boldsymbol{\Delta} \hat{\mathbf{e}}_{s}$ and the optimal velocity $\boldsymbol{\Delta} \hat{\mathbf{e}}_{v}$ error based on the complementary Kalman filter in [17]. In this paper, there are 6 error states for the complementary Kalman filter as show in (8), where $\Delta e_{s, i}$ and $\Delta e_{v, i}$ are the error of estimated position and the error of estimated velocity in $i$-axis of initial frame in order.

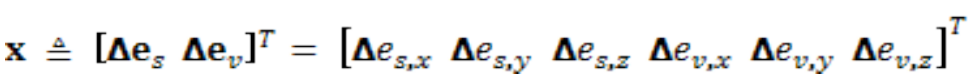

\section{Switching algorithm}

To avoid the inaccuracy of the recognized position $\tilde{\mathbf{s}}$ while the object moving, the switching algorithm will switch the mode of estimation. One of the estimation modes is halt-state which the tracked object is not moving, and another is moving-state that the object is moving. Based on the previous study, the movement of the object is effectively detected by the magnitude of the angular velocity signals, $\Omega$. When this magnitude is equal or greater than the threshold $\varepsilon$ the moving-state mode will operate. Therefore, the switching factor $\rho$ at time $k$ is defined as in Eq (9).

$\rho_{k}=\left\{\begin{array}{l}0, \Omega_{k} \geq \varepsilon \\ 1, \Omega_{k}<\varepsilon\end{array}\right.$

According to the switching algorithm in [11], the equations Eq (3) and Eq (4) are modified as shown in Eq (10) and Eq (11) in order.

$\widehat{\mathbf{x}}_{k}=\mathbf{F} \widehat{\mathbf{x}}_{k-1}+\mathbf{G} \widehat{\mathbf{x}}_{k-1}+\rho_{k} \mathbf{K}_{k}\left(\mathbf{z}_{k}-\overline{\mathbf{z}}_{k}\right)$

$\mathbf{K}_{k}=\rho_{k} \mathbf{P}_{k} \mathbf{H}^{T}\left[\mathbf{H P}_{k} \mathbf{H}^{T}+\mathbf{R}_{k}\right]^{-1}$

Based on the error state in Eq (8), the error measurement vector is the vector that contains the measurement error. The measure position error $\Delta \mathbf{e}_{s, m e a}$ is defined as in Eq (12). While haltstate mode, the measurement bias is the different between the current predicted position $\overline{\mathbf{s}}$ and the current recognized position $\tilde{\mathbf{s}}$.

$\mathbf{z}_{k}=\boldsymbol{\Delta} \mathbf{e}_{s, m e a, k} \triangleq \overline{\mathbf{s}}_{k}-\tilde{\mathbf{s}}_{k}$

\section{Error model formulation}

From Eq (2), the system error dynamics can now be formed as in Eq (13),

$\mathbf{x}_{k}=\left[\begin{array}{l}\boldsymbol{\Delta} \mathbf{e}_{s, k} \\ \boldsymbol{\Delta} \mathbf{e}_{v, k}\end{array}\right]=\mathbf{F}\left[\begin{array}{l}\boldsymbol{\Delta} \mathbf{e}_{s, k-1} \\ \boldsymbol{\Delta} \mathbf{e}_{v, k-1}\end{array}\right]$

where the state transition $\mathbf{F}$ is determined by the propagation model in [17]. 
$\mathbf{F}=\left[\begin{array}{ccc}\mathbf{I} & \vdots & \mathbf{0} \\ \cdots & \cdots & \cdots \\ (-\beta \cdot \tau) \mathbf{I} & \vdots & \mathbf{I}\end{array}\right]$

In Eq (14), $\mathbf{I}$ is a 3-by-3 identical matrix, $\mathbf{0}$ is a 3-by-3 zero matric and $\tau$ is time step size. The time constant $\beta$ is used for parameter optimization. The system error-measurement dynamics can also be formed from (2) as in Eq (15), where the observation matrix $\mathbf{H}$ is defined as in Eq (16).

$\mathbf{z}_{k}=\boldsymbol{\Delta} \mathbf{e}_{s, m e a, k}=\mathbf{H}\left[\begin{array}{l}\boldsymbol{\Delta} \mathbf{e}_{s, k} \\ \Delta \mathbf{e}_{v, k}\end{array}\right]$

$\mathbf{H}=\left[\begin{array}{llllll}1 & 0 & 0 & 0 & 0 & 0 \\ 0 & 1 & 0 & 0 & 0 & 0 \\ 0 & 0 & 1 & 0 & 0 & 0\end{array}\right]$

As the result from above, the modified state prediction equation in Eq (10) will be:

$\widehat{\mathbf{x}}_{k}=\mathbf{F} \widehat{\mathbf{x}}_{k-1}+\rho_{k} \mathbf{K}_{k}\left(\mathbf{z}_{k}-\overline{\mathbf{z}}_{k}\right)$

\section{Experiment}

In this Section, the position estimation module is tested with the measurement signal form IMU attached on a stick as Figure 3 . Before this experiment, the parameters of the error model in the attitude estimation module and the position estimation module are already optimized by simulation with white noise.

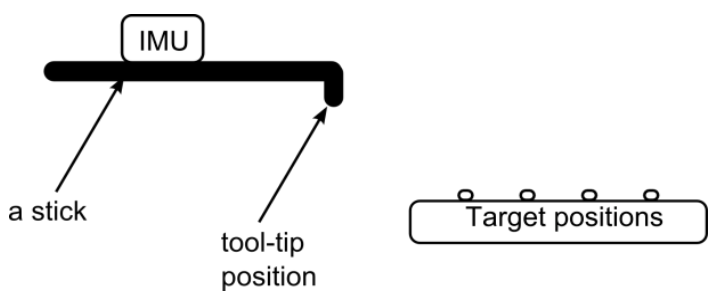

Figure 3: a stick attached with IMU

To test the performance of the position estimation module, the stick is attached with a low-cost IMU. The measurement data from the IMU are acquired by the Labview application which is developed in Competence Center for Applied Sensor Systems (ccass). Moreover, this Labview application determined the arrived target position based on the algorithm of the positionidentification module.

In this experiment, the measurement data are acquired from an IMU100, which is successfully developed by ccass, which is attached on the intelligent screwdriver. The IMU100 is 9-DOF initial measurement unit integrated with 3 -axis magnetometer, a digital thermometer and a 
digital pressure sensor. The 3-axis magnetometer in IMU 100 is LSM303DLH from ST, which give noise $218 \mu \mathrm{m} / \sqrt{\mathrm{Hz}}$ ) and its sensitivity is $\pm 20 \mathrm{mg}$. The gyroscopes of this IMU are LPR430AL and LY330ALH from ST. The rate noise density of LY330ALH is less than LY330ALH (0.014 dps $/ \sqrt{\mathrm{Hz}}$ ) and $0.018 \mathrm{dps} / \sqrt{\mathrm{Hz}}$ ) in order). The reference tracking system is the AS200 infrared tracking system from Lukotronic. And the reference position is measured from the infrared markers.

The experiment was done by moving the stick from initial position (B00) to a target position as in Figure 4 (5 times per target position, and 8 target positions). In detail, the pitch between each target position is $10 \mathrm{~cm}$. After processing the measurement data, the estimated positions were compared with the measured position from the infrared tracking system.

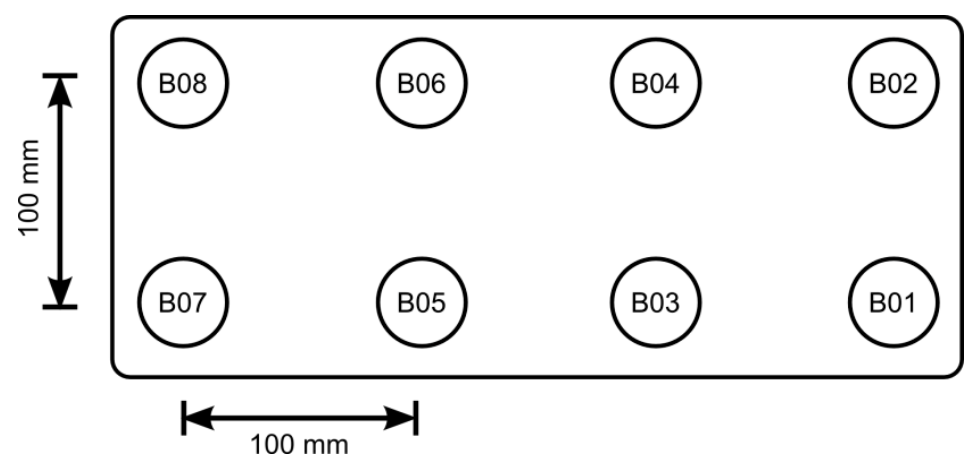

Figure 4: target positions

\section{Results}

The errors of the tool-tip tracking algorithms are analyzed by the target bolt position and the estimated position in each axis. As Figure, the maximum average error of position estimation was $188 \mathrm{~mm}$ in $\mathrm{x}$-axis of the target position B02. The reason that the error of this position is relatively larger than other target positions is the position error of this position is greater than the limit of the position-identification module (within $100 \mathrm{~mm}$ diameter). Therefore, the positionidentification module predicted a false position and over compensated the optimal position in the position estimation module. In summary, the performance of this attitude estimation algorithm is satisfied. The overall average position errors from the experiments are 69,41 and $42 \mathrm{~mm}$. in $\mathrm{x}-, \mathrm{y}$ - and $\mathrm{z}$-axis in order. 


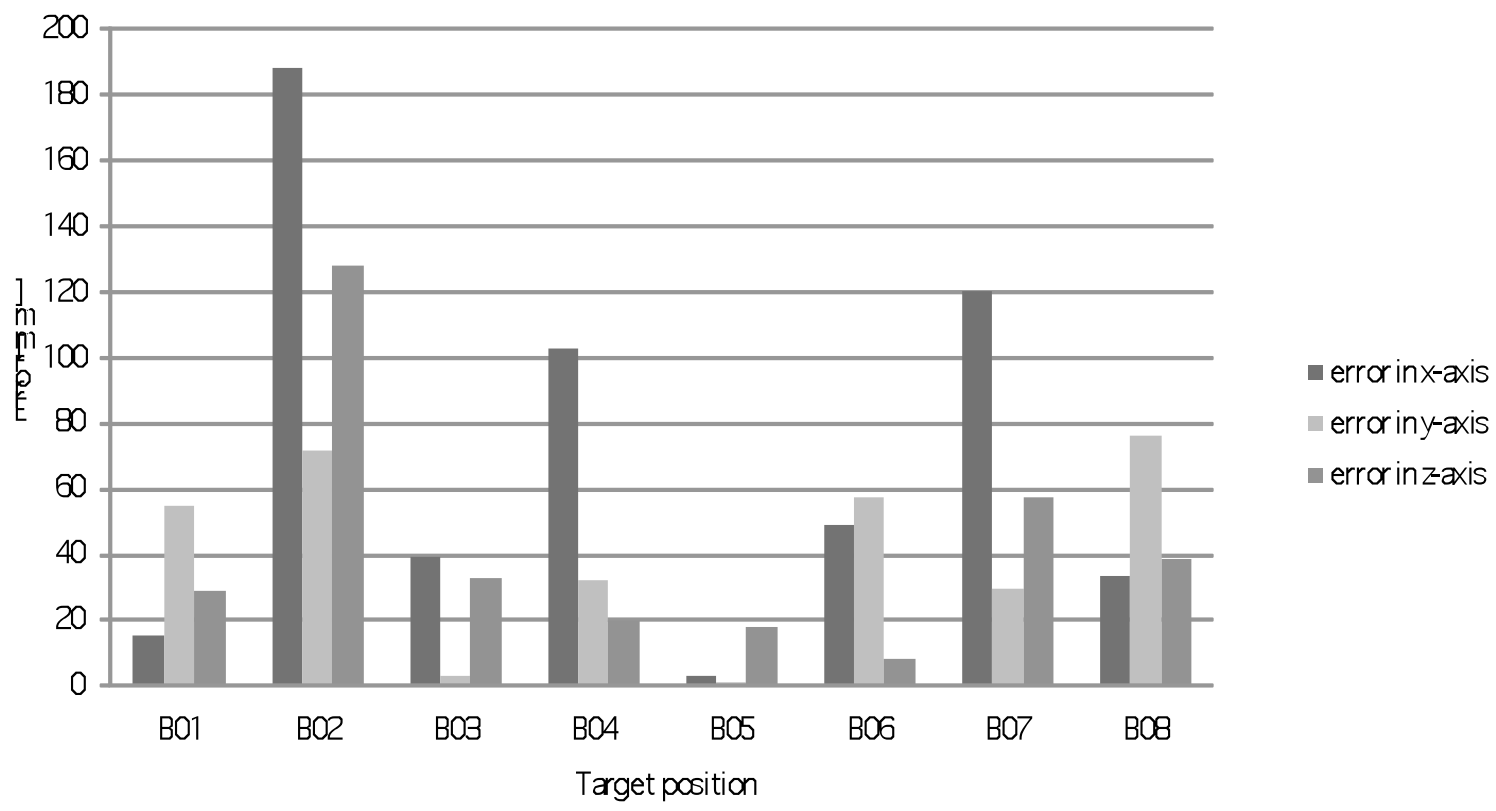

Figure 5: average error of position estimation

\section{Conclusion and outlook}

This paper described a position estimation based on complementary Kalman filter (CKF). This algorithm is part of the project "INSCHRAV" that its target is to develop a tracking system to track an intelligent screwdriver in automotive production line, by using low-cost IMU. With the simplified error model, which is described in this paper, the optimal attitude is able to be successfully computed as the result from the experiment.

To improve the performance of the tool-tip tracking algorithms, the error model based on CKF for attitude estimation and position estimation would be optimized. Moreover, this position estimation with help of pattern recognition algorithms is able to be implemented in other indoor navigations, such as a computer interface devices and video game controllers.

\section{Literatures:}

[1] D. H. Titterton and J. L. Weston, "Strapdown Inertial Navigation Technology," The Institution of Electrical Engineers and The American Institute of Aeronautics and Astronautics, 2004.

[2] M. Haid, T. Dorf, G. Marquardt, and U. Schneider, "Orientation tracking with low cost sensors as a kalman fiter application," IAIN 2003, World Congress, Berlin, 2003.

[3] B. Friedland, "Treatment of bias in recursive filtering," Automatic Control, IEEE Transactions on, vol. 14, no. 4, pp. 359 - 367, aug 1969.

[4] E. Foxlin, "Inertial head-tracker sensor fusion by a complimentary separate-bias kalman filter," Virtual Reality Annual International Symposium, vol. 0, p. 185, 1996.

[5] R. Zhu and Z. Zhou, "A small low-cost hybrid orientation system and its error analysis," Sensors Journal, IEEE, vol. 9, no. 3, pp. 223 -230, March 2009. 
[6] Y. Xiaoping, E. Bachmann, and R. McGhee, "A simplified quaternion based algorithm for orientation estimation from earth gravity and magnetic field measurements," Instrumentation and Measurement, IEEE Transactions on, vol. 57, no. 3, pp. $638-650$, March 2008.

[7] L. Zhao, Q. Nie, and Q. Guo, “Unscented kalman filtering for SINS attitude estimation," in Control and Automation, 2007. ICCA 2007. IEEE International Conference on, 302007-june1 2007, pp. 228 -232.

[8] M. Romanovas, L. Klingbeil, M. Trachtler, and Y. Manoli, "Efficient orientation estimation algorithm for low cost inertial and magnetic sensor systems," in Statistical Signal Processing, 2009. SSP '09. IEEE/SP 15 Workshop on, 312009-sept.3 2009, pp. 586 -589.

[9] P. Martin and E. Salaun, "Invariant observers for attitude and heading estimation from low-cost inertial and magnetic sensors," in Decision and Control, 2007 46th IEEE Conference on, dec. 2007, pp. 1039 -1045.

[10]J. Marins, X. Yun, E. Bachmann, R. McGhee, and M. Zyda, "An extended Kalman filter for quaternion-based orientation estimation using MARG sensors," in Intelligent Robots and Systems, 2001. Proceedings. 2001 IEEE/RSJ International Conference on, vol. 4, 2001, pp. 2003-2011 vol.4.

[11]H. Rehbinder and X. Hu, "Drift-free attitude estimation for accelerated rigid bodies," Automatica, vol. 40, no. 4, pp. $653-659,2004$.

[12]X. Kong, "Ins algorithm using quaternion model for low cost imu,"Robotics and Autonomous Systems, vol. 46, no. 4, pp. $221-246,2004$.

[13]S. Julier, J. UhImann, and H. Durrant-Whyte, "A new approach for filtering nonlinear systems," in American Control Conference, 1995. Proceedings of the, vol. 3, jun 1995, pp. $1628-1632$ vol.3.

[14]W. Dayawansa and C. Martin, "A converse lyapunov theorem for a class of dynamical systems which undergo switching," Automatic Control, IEEE Transactions on, vol. 44, no. 4, pp. 751 -760, april 1999.

[15]P. S. Mayback, Stochastic models, estimation, and control. ACASEMIC PRESS, 1979, vol. I.

[16]O. Loffeld, Estimation Theory II, Kalman-filter Application (Estimationstheorie II, Anwendungen Kalman Filter). Munich, Vienna: R. Oldenbourg-Verlag, 1990.

[17] M. Haid, "Improvement of the referenceless inertial objeckt-tracking for low-cost indoor-navigaton by kalmanfiltering (verbesserung der referenzlosen inertialen objecktverfolgung zur low-cost indoor-navigaton durch anwendung der kalman-filterung)," Ph.D. dissertation, Universitaet Siegen, 2004.

[18]B. Barshan and H. Durrant-Whyte, "Inertial navigation systems for mobile robots," Robotics and Automation, IEEE Transactions on, vol. 11, no. 3, pp. 328 -342, Jun. 1995.

[19] O. Hegrenses, O. Hallingstad, and B. Jalving, "Comparison of mathematical models for the hugin 4500 auv based on experimental data," in Underwater Technology and Workshop on Scientific Use of Submarine Cables and Related Technologies, 2007. Symposium on, april 2007, pp. 558 -567.

[20]Won, S. H., Golnaraghi, F., and Melek, W. (2009), "A fastening tool tracking system using an IMU and a position sensor with Kalman filters and a fuzzy expert system", Industrial Electronics, IEEE Transactions on, vol. 56 , no. 5 , pp. $1782-1792$.

[21]Vissiere, D., Martin, A., and Petit, N. (2007), "Using distributed magnetometers to increase IMU-based velocity estimation into perturbed area", Decision and Control 2007, 46th IEEE Conference, pp. 4924 -4931. 